\title{
Missions militaires italiennes à l'étranger : la prolifération des hybrides
}

\section{Salvatore Palidda}

\section{OpenEdition}

\section{Journals}

Édition électronique

URL : http://journals.openedition.org/conflits/3126

DOI : $10.4000 /$ conflits.3126

ISSN : $1777-5345$

Éditeur :

CCLS - Centre d'études sur les conflits lilberté et sécurité, L'Harmattan

Édition imprimée

Date de publication : 15 novembre 2007

Pagination : 113-129

ISBN : 978-2-296-04582-8

ISSN : 1157-996X

Référence électronique

Salvatore Palidda, «Missions militaires italiennes à l'étranger : la prolifération des hybrides », Cultures \& Conflits [En ligne], 67 | automne 2007, mis en ligne le 04 janvier 2010, consulté le 30 mars 2021. URL : http://journals.openedition.org/conflits/3126 ; DOI : https://doi.org/10.4000/conflits.3126 


\section{Missions militaires italiennes à l'étranger : la prolifération des hybrides}

\section{Salvatore PALIDDA}

\section{Salvatore Palidda est professeur de sociologie à l'université de Gênes.}

T es missions militaires italiennes à l'étranger au cours de ces dernières Lannées peuvent être considérées comme un « fait politique total». Elles sont en effet caractérisées et marquées par l'entrelacement de multiples aspects et acteurs de la société italienne ainsi que de leurs relations avec l'extérieur. Elles sont ainsi révélatrices des mutations économiques et politiques que ce pays a connues depuis la fin des années 1970 . Nombre de ces aspects et acteurs, ainsi que les multiples interactions en jeu (directes et indirectes), ne sont pas évidents à analyser, les éléments empiriques nécessaires à une étude sociologique approfondie n'étant pas toujours disponibles ${ }^{1}$. En effet, il s'agit d'un univers d'activités qui oscillent entre le public et le secret, et parfois même entre le légal et l'illégal (voir infra). Par ailleurs, les perspectives interprétatives et d'analyse pour la recherche dans ce domaine ne semblent pas encore suffisamment adaptées aux changements qui se sont succédés depuis le début des années 1980 et davantage encore au cours de ces dernières années. En effet, les conséquences de la «Révolution dans les affaires militaires" (RAM ${ }^{2}$ ), de la révolution technologique et du développement néo-libéral ${ }^{3}$ ont provoqué une prolifération et une hybridation de différents éléments et acteurs qu'il n'est pas toujours facile d'appréhender au travers d'un cadre d'analyse unitaire ${ }^{4}$. Cela est d'autant plus vrai que, si le développement des activités économiques et surtout financières des acteurs italiens à l'étranger sont apparemment tout à fait indépendantes des affaires militaires, les choix

1. Il est ainsi impossible d'établir un recensement de toutes les activités et acteurs italiens à l'étranger car de nombreuses délocalisations passent par des sociétés ayant leur siège dans des paradis fiscaux ou qui sont tout à fait illicites du point de vue du droit italien. Cela vaut aussi pour les «security contractors» italiens.

2. Voir à ce propos Wasinski C., «Créer une Révolution dans les affaires militaires : mode d'emploi », Cultures E Conflits, n64, 2006, pp. 149-164.

3. Voir les articles de A. Dal Lago et de A. Joxe dans Conflitti Globali, n¹/2005.

4. Voir Un mondo di controlli, Conflitti Globali, $\mathrm{n}^{\circ} 5 / 2007$. 
dans ce dernier domaine semblent pourtant de plus en plus conditionnés par les premières (et cela ne concerne pas seulement le marché des armements).

Nous allons ici essayer de fournir l'essentiel des connaissances acquises et des pistes que notre recherche - qui en est encore à ses débuts - poursuit.

\section{La découverte des missions militaires extérieures par l'Italie}

Rappelons tout d'abord qu'après la Seconde Guerre mondiale, la souveraineté italienne dans le domaine militaire a été subordonnée à celle des EtatsUnis (directement et au travers de l'OTAN) ${ }^{5}$. Il suffit ici de rappeler que toutes les bases militaires américaines et otaniennes en Italie sont soumises à l'hégémonie de la hiérarchie militaire américaine, notamment en ce qui concerne le stockage et l'emploi éventuel d'armes atomiques, des systèmes d'armement stratégiques, du contrôle des communications et de l'espace et même en ce qui concerne la conduite des militaires américains hors des bases 6 .

Cependant, la Charte constitutionnelle de la République née de la résistance au nazisme et au fascisme a établi des normes plutôt progressistes et pacifistes. En effet, l'article 11 affirme :

«L'Italie répudie la guerre en tant que moyen d'affront à la liberté des autres peuples et comme moyen de solution des différends internationaux ; elle consent, dans des conditions de parité avec les autres Etats, aux limitations de souveraineté nécessaires à un ordre assurant la paix et la justice entre les nations ; elle promeut les organisations internationales poursuivant ces buts ».

Les interprétations de cet article ont suscité de nombreuses critiques à l'égard du déploiement sur le sol italien d'armes, de forces et de systèmes d'ar-

5. Sur l'histoire de la souveraineté italienne, voir Palidda S., «L'Anamorphose de l'Etat-nation: le cas italien ", Les Cabiers internationaux de sociologie, n' 93 , 1992, pp. 269-298. L'une des raisons principales de dissension entre l'Italie et les Etats-Unis a toujours été la tentative italienne de jouer un double jeu : fidèle allié subalterne d'un côté, marchand autonome de l'autre. Enrico Mattei, le patron de l'ENI, dans l'après-guerre, fut éliminé parce qu'il affirmait une trop grande autonomie, réussissant à tisser des relations privilégiées avec nombre de pays arabes, parfois en échange d'armes (notamment au FLN algérien, et aux Palestiniens). De la même façon, la " guerre américaine » contre la mafia (l'affaire Sindona, l'Opus Dei, puis les attaques contre Andreotti) semble s'inscrire dans le cadre d'une tentative de la part du gouvernement américain de reprendre le contrôle sur d'anciens «sous-traitants » désireux de s'autonomiser ou de se faire payer davantage (voir par exemple le cas Noriega).

6. La liste des éléments qui montrent ce caractère subalterne de l'Italie est longue. Parmi les derniers faits en date, rappelons l'utilisation de bases pour les « vols de la CIA » qui ont fait l'objet d'enquêtes du Parlement européen et du Conseil de l'Europe. Il est vrai que cela concerne également les bases américaines en Allemagne, mais il faut rappeler que, depuis 1979, le territoire italien a été considéré comme une sorte d'avant-poste indispensable pour la projection vers les fronts sud et sud-est (depuis le déploiement des euromissiles en Sicile, puis le développement très important - encore en cours - des bases américaines sur cette île). 
mes destinés à des actes de guerre contre d'autres Etats. Les manifestations contre les accords de défense engageant l'Italie dans des orientations stratégiques à l'extérieur, contre les missions des forces militaires nationales à l'étranger ou encore contre l'industrie militaire n'ont cessé depuis la Guerre froide 7.

En effet, le premier aspect singulier du cas italien concerne l'importance de sa production et de son exportation d'armes, qui la place parmi les cinq premiers Etats du monde dans ce domaine ${ }^{8}$. Or, cette réussite relève à la fois des intérêts de l'industrie italienne et de l'étroite dépendance vis-à-vis des Etats-Unis (qui sont le premier pays importateur d'armements italiens, avant les Emirats Arabes Unis et le premier fournisseur de l'Italie). Elle est également à mettre en relation avec l'engagement italien dans les missions militaires voulues par les Etats-Unis, ainsi que par certains lobbies italiens, notamment pour maintenir et gagner des marges de marché, pour protéger les délocalisations des activités économiques et, donc, pour ne pas rester en marge des bénéfices que le nouveau « keynésianisme militaire » peut assurer aux différents acteurs concernés 9.

7. Rappelons que l'Italie a eu un puissant mouvement pour la paix dès les années 1950 sur l'initiative de catholiques et de la gauche. Par ailleurs, toutes les tentatives graves de «coups » autoritaires ont été directement liées aux milieux les plus réactionnaires des Etats-Unis et des forces armées (la littérature sur cet aspect est assez vaste. Voir notamment Ambrogio V., I servizi segreti italiani, Rome, ADN Kronos, 1985 ; De Lutiis G., I servizi segreti in Italia, Rome, Editori Riuniti, 1998 ; Galli G., Piombo rosso. La storia completa della lotta armata in Italia dal 1970 a oggi, Milan, Baldini Castoldi Dalai, 2007). Beaucoup de pacifistes ou de « neutralistes » italiens sont particulièrement attentifs au budget du secteur de la production et du commerce d'armements (voir en particulier le site http://www.sbilanciamoci.org/). En revanche, peu d'attention est portée dans la recherche sur la dimension économique de la sécurité intérieure.

8. Selon la Sipri YearBook, l'Italie était au septième rang mondial en matière de transfert d'armements dans la période 1999-2003 mais depuis, elle semble se placer entre la quatrième et la cinquième place. Finmeccanica se situe parmi les dix premières entreprises d'armes mondiales (qui sont toutes américaines, sauf une anglaise et une française). Voir La Pira G., Il Commercio Delle Armi. L'Italia nel contesto internazionale, Annuario Armi-Disarmo, Milano, Jaca Book, 2004. L'industrie militaire italienne - déjà importante lors de la Première Guerre mondiale et pendant le fascisme, à l'instar des autres principaux pays européens - a connu un développement considérable depuis la Seconde Guerre mondiale (voir Battistelli F., Armi : nuovo modello di sviluppo?, Turin, Einaudi, 1982). Elle maintient une position de premier plan, non seulement dans les armes légères (pistolets et fusils Beretta, etc.) et les mines parfois vendues illégalement à travers le monde, mais aussi dans les armements de pointe, voire à très haute technologie. Les derniers succès commerciaux concernent en particulier une flotte d'hélicoptères pour le président des EtatsUnis (premier pays importateur d'armements italiens, suivi par les Emirats arabes unis, la Pologne et le Royaume-Uni). Les principaux fournisseurs d'armements à l'Italie sont les EtatsUnis, la Suisse et la Fédération russe (voir http://www.difesa.it et http://www.disarmo.org).

9. Remarquons qu'aujourd'hui, les parlementaires les plus engagés dans le soutien à la cause de l'industrie militaire italienne sont ceux des démocrates de gauche (ex-parti communiste). Ainsi, en 2007, l'actuel sous-secrétaire à la Défense Forcieri a signé à Washington l'accord pour le bouclier spatial et pour la fabrication en Italie des avions de combat à portée stratégique sans consultation du Parlement ou même du Conseil des ministres (mais en accord avec le chef du gouvernement et le ministre des Affaires étrangères, D’Alema - lui aussi un ancien du PCI - qui, semble-t-il, ambitionne le poste de Secrétaire général de l'OTAN). L'un des faits illicites les plus ignorés concerne le passage direct de nombre de hauts gradés des forces armées à des postes de direction de l'industrie militaire, ce qui est interdit par la loi. Il faut également noter qu'une importante partie de cette industrie s'est convertie à la production de systèmes de sécurité urbaine dont la vidéo-surveillance, avec la collaboration active de certains départements universitaires. 
Les Italiens ont souvent été décrits comme des « marchands levantins » capables de faire du bachich avec ceux que les autres «Occidentaux » considèrent pourtant comme leurs pires ennemis ${ }^{10}$. En effet, les entreprises et hommes d'affaire italiens semblent souvent privilégier la logique du profit sur tout autre considération. Bien évidemment, aucun acteur italien n'ose s'imaginer agir contre l'avis de l'administration américaine ; mais, dans le même temps, ils recherchent toujours des marges de manœuvre autonomes. Ainsi, les services secrets italiens ont toujours cherché à concilier fidélité à l'allié-dominant et ententes occasionnelles avec le reste du monde, suscitant parfois des réactions violentes de la part des alliés. A ce propos, un des épisodes les plus graves semble avoir été celui de l'assassinat, par des militaires américains, du fonctionnaire des services secrets italiens Calipari, lors de la libération de la journaliste Sgrena le 4 mars $2005{ }^{11}$.

\section{Les changements dans les missions militaires}

L'histoire des missions militaires italiennes à l'étranger est assez récente : depuis le début des années 1980, on en compte environ soixante, entre les missions dites d'entraînement, d'observation, de secours humanitaire et de sécurité internationale («maintien de la paix » et «imposition de la paix »-peacekeeping et peace-enforcement - selon la terminologie édulcorée ${ }^{12}$ ). En réalité, les missions considérées comme les plus importantes du point de vue des militaires sont les vingt-deux missions qui ont eu lieu ces quinze dernières années, dont onze dans les pays de l'Europe du Sud-Est (notamment dans les Balkans) ${ }^{13}$.

10. Voir Dal Lago A., «Esistono davvero i conflitti tra culture ?», in Galli C. (a cura di), Multiculturalismo, Bologna, il Mulino, 2006.

11. Le cas de l'attaque américaine qui a provoqué la mort de Calipari fait encore l'objet d'une enquête judiciaire que les Etats-Unis refusent catégoriquement d'accepter (tout comme pour le procès contre vingt-trois agents de la CIA pour l'enlèvement et la déportation d'Abu Omar). Selon la reconstruction proposée par nombre d'experts (dont les journalistes D’Avanzo et Bonini, l'historien des services secrets Giannuli et quelques parlementaires), le conflit entre l'Italie et les Etats-Unis portant sur la conduite à adopter lors d'enlèvements d'Italiens en Irak a éclaté à l'occasion de la libération de Sgrena : c'était la quatrième fois que les services italiens agissaient à l'encontre des directives américaines. Ainsi, presque tous les analystes italiens considèrent comme évident que l'assassinat de Calipari (Sgrena elle-même et un autre agent italien ont été blessés) a constitué le « coup de frein » de la part de l'administration américaine aux comportements par trop autonomes des Italiens (bien représentés par l'action de ce fonctionnaire dont la femme est actuellement député au titre de la coalition de gauche). Olimpio G., Operazione Hotel California, Milan, Feltrinelli, 2005 ; Bonini C., D’Avanzo G., Il mercato della paura. La guerra al terrorismo islamico nel grande inganno italiano, Turin, Einaudi, 2006; Malabarba G., 2001-2006 segreti e bugie di stato, Roma, Liberazione/Alegre, 2006 ; Giannuli A., op. cit.

12 . Nous reprenons ici et par la suite des termes anglais puisqu'en Italie, ils sont désormais courants dans le langage des militaires, mais aussi dans celui des hommes politiques, des journalistes et même des dissidents. C'est assez révélateur de l'influence exercée, à droite comme à gauche, par les Américains et les Anglo-saxons de manière plus générale. Il est évident que, depuis le succès de l'idée de " guerre humanitaire ", nombre d'oxymores décrivent à la fois les missions militaires et leur liens avec les activités des ONG qui les accompagnent au nom de l'Etat, mais parfois aussi au nom du pacifisme.

13. Missions dans le cadre de l'ONU (UNIFIL au Liban en 1979, Namibie en 1989-1990, Somalie en 1992-1994, Mozambique en 1993-1994, Soudan en 2005) ; missions multinationales (Libano 1 et 2 en 1982-1984, Kurdistan en 1991, Rwanda en 1994, Albanie Alba en 1997, 
Rappelons d'abord que le changement du «modèle de défense » italien débute à la fin des années 1970, sous l'influence américaine et sous celle d'un nouveau lobby militaro-industriel italien ${ }^{14}$. Il s'accélère avec la prolifération des opérations militaires extérieures des pays les plus industrialisés ${ }^{15}$. Les tâches ou missions des forces armées ayant été définies officiellement vont bien au-delà de ce que prescrit la Charte constitutionnelle (que l'on pourrait décrire comme strictement défensive). Par exemple, selon le chef d'état-major des forces armées, le concept ou principe de défense des intérêts vitaux du pays, quelque soit l'endroit où ils sont menacés, s'étend aussi à :

« la sauvegarde des espaces euro-atlantiques; la contribution à la gestion de crises internationales au travers de la participation à des opérations de prévention et de gestion de crise, afin de garantir la paix, la sécurité, la stabilité et la légalité internationale; [...] l'affirmation des droits fondamentaux de l'Homme, dans l'esprit de la Charte des Nations unies, dans le cadre d'organisations internationales - tout d'abord l'OTAN, l'UE et l'ONU - et/ou d'accords bi- ou multilatéraux [...]; le concours à la sauvegarde de la liberté des institutions et la réalisation de tâches spécifiques dans des circonstances de calamité publique et dans d'autres cas de nécessité extraordinaire et urgente 16 ».

En outre, la directive ministérielle mentionne comme élément commun à ces quatre types de missions :

«l'exigence prioritaire d'étendre l'action de l'instrument militaire aux plus récentes formes de conflit asymétrique, avec une attention particulière à la lutte contre le terrorisme international et la prolifération des armes de destruction massive. Au plan conceptuel, les quatre missions ne doivent pas êtres considérées séparément, mais dans une optique intégrée, puisqu'elles contribuent en synergie à garantir la sécurité et la défense de l'Etat ».

Timor oriental en 1999-2000, Afghanistan en 2002-2003, Irak Antica Babilonia en 20032006) ; missions de l'OTAN (Bosnie en 1995-2004, Kosovo en 1998, Albanie en 1999, Macedonia Essential Harvest en 2001, Macedonia Amber Fox en 2002-2003, Afghanistan depuis 2003). La première mission importante a eu lieu en 1982 au Liban (2 000 soldats; en deux ans plus de 8000 et, au total, 1 mort et 75 blessés). La mission Pellicano en Albanie (16 septembre 1991 - 3 décembre 2003) a été la seule qui ait entièrement été programmée et réalisée par l'Italie en dehors de tout mandat multilatéral.

14. Remarquons entre autres que les dirigeants des industries militaires italiennes sont des grands commis d'Etat puisque ces industries sont, dans la plupart des cas, publiques. Depuis les années 1970 y figurent des homini novi issus des milieux de la gauche et des généraux à la retraite (parfois anticipée à 60 ans) bien que, comme nous l'avons déjà vu, la loi italienne interdise le passage direct de la hiérarchie militaire à la direction de l'industrie militaire, ce que presque personne n'a jamais commenté.

15. Voir Palidda S., 1991, op. cit.

16. Voir Di Paola G., chef d'état-major, Il Concetto Strategico del Capo di Stato Maggiore della Difesa, 2007 : www.difesa.it/NR/rdonlyres/7CF00FEA-D74E-4533 B3ED9CCB3ED9CC CD8B79E89/0/libroconcettostrategico.pdf 
«Les nouvelles technologies peuvent révolutionner les formes de la compétition politique, économique et militaire... Existent aussi des facteurs potentiels de déstabilisation liés à des phénomènes de nature politico-sociale comme l'immigration ou les trafics illégaux... [cela implique nécessairement des] changements des forces et des doctrines d'emploi, surtout en termes de flexibilité et de capacité diversifiée de réaction... ${ }^{17}$ ».

En décryptant cette définition des missions des forces armées italiennes, on peut y trouver l'objectif de défendre les intérêts du pays partout dans le monde. Cette tâche est étroitement liée au respect des engagements vis-à-vis des alliés, notamment au nom des valeurs occidentales. Dans les faits, il découle de cet élargissement considérable du mandat de forces armées un champ d'action accrue pour les ONG, mais aussi pour des pratiques de sous-traitance à des entreprises privées, lesquelles pratiques sont jugées indispensables à la «flexibilité » et la «diversification de la réaction », surtout face à une menace comme le terrorisme. L'action militaro-humanitaire n'est alors plus une simple facette paternaliste ou une «tactique » de " protection de la force » en mission extérieure ${ }^{18}$. C'est aussi le biais qui permet de promouvoir les projets intéressants, les entreprises italiennes et le commerce d'armement. En effet, surtout au cours des années 1990, de nombreuses activités économiques italiennes ont été délocalisées partout dans le monde, et en particulier vers des «zones à risque » (Moyen-Orient, Golfe, Afrique et Amérique latine). Bien que l'univers social complexe qui résulte de la convergence de ces évolutions soit peu étudié, les rares recherches qualitatives semblent montrer qu'il implique des acteurs divers et variés, s'engageant occasionnellement ou durablement dans des activités étroitement interdépendantes: entrepreneurs, financiers, médiateurs, entreprises de sous-traitance, employés d'ONG, militaires, policiers, mais aussi des personnes liées au milieux du crime ${ }^{19}$. En Albanie, en Roumanie, dans le Maghreb, au Niger, et en Somalie notamment,

17. Il est rare que l'on parle explicitement de problèmes de protection des activités économiques italiennes à l'étranger, alors que le problème est assez connu (par exemple dans le cas de la base de Nassiryia très proche des sites de l'ENI). Depuis environ quinze ans, la hiérarchie militaire italienne a développé une sensibilité particulière pour les études stratégiques, encore qu'on ne peut pas dire qu'il y ait des militaires intellectuels comparables à ceux qu'ont connus les autres pays. Certains d'entre eux ont fait des propositions allant dans le sens de l'emploi des militaires à la fois dans la sécurité intérieure, dans la lutte contre la criminalité organisée et dans la « guerre » aux migrations clandestines souvent sous couvert de « guerre aux trafiquants ».

18. Les ONG peuvent entretenir des contacts avec l'ennemi, et même amorcer des négociations au cas par cas, encore qu'elles prennent le risque d'être à la merci de cet ennemi. Cela est arrivé à plusieurs reprises avec pour conséquence des enlèvements de représentant d'ONG et la mort de trois d'entre eux en Irak et en Afghanistan.

19. Nous faisons ici allusion aux nombreuses observations faites par des chercheurs et des employés d'ONG en Albanie, Roumanie, Maghreb, Nigéria, Niger, Irak et Afghanistan. Voir Palidda S., European Activities Delocalisation in Third Countries, Human Rights and Neocolonialism Aspects, Challenge Working Paper, mars 2008. 
les entreprises italiennes ont parfois accepté l'offre de prestations de la part de médiateurs ambigus, la protection des entreprises de sécurité privée, des militaires et des policiers italiens en opération extérieure tout en nouant des ententes avec les ONG présentes sur place. Rappelons que l'un des cas les plus éclatants de la «mauvaise tournure » que peut prendre ce type d'interrelations est celui de la mission «Arcobaleno » en Albanie, dont nombre d'acteurs sont encore en procès ${ }^{20}$. Par ailleurs, certains militaires - $\mathrm{y}$ compris des officiers - ont dénoncé le fait que, pour s'assurer une mission à l'étranger, ils ont dû donner un mois ou deux de leur salaire - et parfois plus - aux personnes chargées du recrutement ${ }^{21}$.

Pour s'adapter à ces «nouvelles missions ", les forces armées italiennes ont entamé un important processus de modernisation auquel est venu répondre le développement de l'industrie militaire du pays. Dans ce contexte, il faut rappeler la suspension puis l'abolition du service militaire obligatoire 22 (décisions du 8 mai 2001 du gouvernement de centre-gauche puis de celui de centre-droite en août 2004, et enfin du 30 juin 2005). Depuis le $1^{\text {er }}$ janvier 2005, le recrutement se fait ainsi uniquement sur une base volontaire et à caractère professionnel. En effet, les militaires envoyés en mission à l'étranger ont toujours été des volontaires et des professionnels de carrière.

Il est aussi important de souligner qu'au terme de leur contrat de trois ans, ces volontaires sont privilégiés dans le recrutement au sein des forces de police de l'Etat, à moins qu'ils ne soient attirés par les sociétés militaires privées (SMP) ou les entreprises de sécurité privées (SSP) ${ }^{23}$ qui, parfois, recrutent des militaires de carrière et des policiers quittant leurs corps d'origine pour gagner davantage ou qui sont à la recherche d'expériences nouvelles ${ }^{24}$. Par consé-

20. Voir Ciccarelli R., Foglio G., «L'etica ambigua degli aiuti. Il lavoro umanitario fra civile e militare dalle crisi iugoslave alla guerra in Iraq ", in La guerra dei mondi, Conflitti Globali, $\mathrm{n}^{\circ} 1 / 2005$, pp. $160-174$.

21. Voir les lettres de ces militaires sur le site :

http://www.osservatorioiraq.it/modules/wfsection/article.php?articleid=1112 ; elles font l'objet d'une enquête judiciaire en cours.

22. La Charte constitutionnelle définissait «la défense de la Patrie et le service militaire obligatoire » comme un «devoir sacré».

23. Comme nous avons pu le constater au cours de notre recherche (encore en cours) en Italie, beaucoup de SMP et de SSP sont parfois nées ou ont été " générées » par des sociétés de gardiennage ou de police privée. Il ne faut pas oublier que l'Italie a été le pays européen le plus touché par le phénomène des enlèvements et de la criminalité organisée de type mafieux et la demande de gardes du corps et de gardiennage en général a toujours été assez importante, faute de compromis avec le milieu de la criminalité. La majorité de ces sociétés a cherché à pôsséder un siège à l'étranger, notamment dans des paradis fiscaux. Par ailleurs, certaines sociétés sont sous-traitantes pour des compagnies étrangères. De même, nombre de contractors individuels italiens cherchent à être embauchés directement par les sociétés américaines ou britanniques.

24. Voir les articles publiés dans La metamorfosi del guerriero, Conflitti Globali, $\mathrm{n}^{\circ} 3 / 2006$. Selon les parlementaires Agnoletto et Malabarba, des militaires italiens auraient pu interroger des prisonniers dans la prison de Guantanamo. Par ailleurs, des contractors italiens employés par une compagnie américaine auraient été impliqués dans les faits de torture à Abou Ghraib (question parlementaire de six députés à la suite de l'entretien vidéo du 22 février 2006 par Rai Neres 24 de Ali Shalal al Kaisi, l'homme en cagoule détenu à Abou Ghraïb, dont la photo 
quent, les personnels des forces armées, des polices d'Etat, des polices locales (municipales), des polices privées et parfois également des ONG ont un profil sociologique relativement semblable, ce qui contribue à l'hybridation entre militaire et policier ainsi qu'entre public et privé 25 .

Remarquons également que les missions militaires à l'étranger comprennent toujours des personnels de plusieurs structures spéciales des forces armées et des polices, dont les dirigeants ${ }^{26}$ entretiennent souvent des relations étroites avec les ONG ainsi qu'avec les autres Italiens présents sur les théâtres d'opération ${ }^{27}$. Tous ces acteurs sont généralement bien connus et parfois en contact avec les agents des services de renseignement italiens ainsi qu'avec les journalistes des médias italiens (qu'ils soient « embedded» ou non).

Ces interrelations et interactions ne signifient cependant pas que l'on puisse parler d'une «communauté d'intérêts » homogène: des tensions et des luttes, d'ordre bureaucratique, professionnel ou autre, sont en en effet fréquents. Ainsi, officiellement, les gouvernements de centre-gauche et de centre-droit qui se sont succédés ont toujours affirmé que les missions italiennes ont été menées, ou sont encore en cours, en vertu de décisions internationales reconnues par tous les pays démocratiques et que, en tout état de cause, elles ne sont absolument pas des "opérations de guerre ", d'" occupation», d'" agression» ou de «conquête». Autrement dit, elles seraient toujours des "missions de paix», excluant tout engagement direct, autre que défensif, contre un ennemi quelconque. Or, ces déclarations adressées au Parlement et au pays ont toujours été ouvertement démenties dans les récits et écrits des personnels engagés eux-mêmes, y compris par les plus hauts gradés qui revendiquent les moyens nécessaires à des «missions de guerre » qui soumettent les engagés à des risques d'autant plus grands que l'on prétend qu'il s'agit d' « opérations de paix». Ainsi, au cours de l'été 2007, de nombreux militaires italiens ont dénoncé l'accroissement des risques en Afghanistan

avec les bras liés par des fils électriques a fait le tour du monde. Ali Shalal a affirmé que des contractors italiens auraient participé aux tortures et que des Italiens auraient dérobé des objets archéologiques.

25. Ce même type d'observation a également pu être fait pour le cas des Etats-Unis. Voir : Makki S., «Militarisation de l'humanitaire et privatisation du militaire », Cabiers d'études stratégiques n³6-37, Le Débat stratégique américain 2003-2004, Paris, CIRPES, novembre 2004. Sur les relations entre le public et le privé, voir également : « Les entreprises de coercition para-privées. De nouveaux mercenaires ? ", Cultures \& Conflits, n 52, 2003.

26. Par exemple, l'unité de parachutistes « Folgore », le « Tuscania », les GIS (têtes de cuir) ou encore d'autres structures constituent toujours celles qui sont considérées comme étant « de pointe » car, entre autres, liés aux services secrets.

27. Voir Ciccarelli R., Foglio G., op. cit. Sur les ONG embedded, voir Pandolfi M., « La zone grise des guerres humanitaires », Anthropologica, n48, 2006 ; Pandolfi M., «Une souveraineté mouvante et supracoloniale », Multitudes, 2000. Sur les entreprises de sécurité privée et les ONG, voir Giannuli A., "Guerra finanziaria e intelligence ", Un mondo di controlli, Conflitti Globali, ${ }^{\circ} 5 / 2007$, pp. 114-130. Rappelons que la Croix-rouge italienne est une institution constituée en partie de civils et surtout de militaires (c'est l'un des objets controversés de l'assise institutionnelle italienne de l'après-guerre). Par ailleurs, comme le signale Giannuli, depuis longtemps, nombre d'ONG italiennes ont recours à la protection d'entreprises de sécurité privée. 
considérés comme liés à la conduite trop agressive et « guerrière » des alliés américains vis-à-vis des civils afghans (le ministre de la Défense italien et le gouvernement afghan l'ont également regretté) ${ }^{28}$. Remarquons à ce propos que le personnel militaire italien de ces missions est soumis au code militaire de guerre, et non de paix, et que l'une des propositions de loi du gouvernement Berlusconi prévoyait même d'imposer ce code à tout Italien présent dans des zones de conflit - y compris les journalistes en Irak ou en Afghanistan ${ }^{29}$.

\section{Les performances des carabiniers}

L'Arma dei carabinieri (l'équivalent de la gendarmerie française, mais avec un poids relatif significatif à tout point de vue) est à la fois l'une des plus importantes forces de police pour la sécurité intérieure et une des forces armées avec davantage de poids, en particulier et de plus en plus dans le cadre des opérations extérieures. Avec le gouvernement D’Alema, les carabiniers sont devenus une force armée à part entière, c'est-à-dire avec le même statut que l'armée de terre (dont ils faisaient auparavant partie), l'aviation et la marine ${ }^{30}$. Les carabiniers ont des effectifs, des moyens et des structures plus importants que la police d'Etat et que les trois autres forces militaires ${ }^{31}$.

Sur le site Internet de cette armée ${ }^{32}$, les prestations qu'elle a fournies dans le cadre des missions extérieures sont mises en avant pour attester de sa contri-

28. Parmi les rares voix militaires qui se sont ouvertement et librement prononcés sur les missions, citons le général Fabio Mini (http://www.paginedidifesa.it/2006/pdd_060380.html et http://www.peacereporter.net/dettaglio_articolo.php?idc=0\&idart=7603).

29. Le code de guerre prévoit que tout acte illicite, même mineur, doit être jugé par un tribunal militaire et selon le code de la guerre. Voir De Fiores C., L'italia ripudia la guerra?, Roma, Ediesse-Crs, 2002 ; voir aussi les documents sur le site:

http://www.domenicogallo.it/index.asp

30. Ce fait a suscité nombre de protestations de la part des fonctionnaires de la police d'Etat et d'un grand nombre de personnalités de gauche et même du centre, ainsi que d'un courant d'opinion qui a toujours soupçonné cette armée de nourrir une vocation réactionnaire. En effet, ce genre de vocation est présent dans toutes les polices et forces armées italiennes ainsi que dans les polices privées et les entreprises militaires et de sécurité privées souvent liées au milieu de la droite. Ceci dit, il est assez peu probable que cette tendance réactionnaire puisse conduire les carabiniers et des composantes d'autres forces à des projets d'instauration d'un régime policiaro-militaire, parce que, d'une part il n'y en a pas la nécessité dans la mesure où les gouvernements successifs n'ont presque jamais remis en question les attentes conservatrices (fortement soutenues aussi par la hiérarchie de l'église catholique); d'autre part, les politiciens italiens de droite comme de gauche n'ont jamais déçu les revendications des polices et des forces militaires. Dans le choix de D'Alema, il y a eu à la fois l'illusion de capter la confiance des carabiniers vis-à-vis de la gauche, la volonté de faire plaisir à l'administration américaine mais aussi la volonté de promouvoir une force qui incarne l'hybride militaro-policier.

31. En Italie, les forces de police de l'Etat sont les suivantes : la police d'Etat, les carabiniers, la garde des Finances, la garde de Forêts et la police pénitentiaire (plus les douanes, la garde côtière et la garde des Ports). A ces forces s'ajoutent les polices dites locales (c'est-à-dire municipales ou départementales) qui dépendent de chaque collectivité locale et qui au total comptent autant d'effectifs que la police d'Etat, c'est-à-dire environ 120000 hommes et femmes). Quant aux polices privées, elles ont connu un développement assez important arrivant à un nombre d'effectif probablement égal à celui de la police d'Etat.

32 . http://www.carabinieri.it/Internet/Arma/Oggi/Missioni/1936+-+2001/Parte+II/1992+-+1995/ 
bution au « rayonnement » du pays à l'étranger, montrant ainsi que ce sont désormais les opérations extérieures qui constituent le vecteur de légitimation principal de l'institution autant que les missions de sécurité intérieure, dont notamment celles du Reparto Operazioni Speciali ${ }^{33}$. Par ailleurs, le corps des carabiniers se prévaut du plus grand nombre de morts dans le cadre des missions à l'étranger (dont ceux de l'attentat contre le quartier général des carabiniers à Nassiriya, la capitale de la province de Dhi Qar en Irak, en novembre 2004, qui fit 19 victimes italiennes dont 12 carabiniers). L'engagement dans les missions internationales a ainsi considérablement accru le poids institutionnel et symbolique des carabiniers, par rapport à celui des autres forces armées et de police. C'est par ce biais qu'ils ont réussi à s'imposer comme l'outil indispensable et incontournable pour assurer au pays le rang auquel il prétend sur la scène internationale. C'est aussi par ce biais que cette armée a pu nouer des relations plus étroites avec les forces armées et les services de renseignement de l'allié-dominant et des autres alliés, et ce avec le concours du service de renseignement militaire italien (le Sismi ${ }^{34}$ ), généralement très actif dans les opérations extérieures. Ainsi on peut constater que l'engagement des carabiniers (en particulier les unités spéciales tel que le bataillon parachutiste «Tuscania ») dans les missions à l'étranger est à la hauteur de leur quasimonopole sur les opérations antiterroristes en Italie (presque toutes réalisées par le ROS ${ }^{35}$, l'unité spéciale des carabiniers, souvent sur l'impulsion des services secrets américains, encore qu'elles se sont généralement révélées des opérations menées - assez maladroitement il faut le dire - contre des immigrés qui n'avaient rien à voir avec le terrorisme islamiste ${ }^{36}$ ).

Cependant, les opérations extérieures ont également donné lieu à un certain nombre de «bévues » et de «bavures » de nature à jeter un certain discrédit sur l'institution. Ainsi les premières missions militaires importantes des carabiniers ont eu lieu en Somalie dans le cadre de l'opération «Restore Hope » ( «redonner espoir ») qui - triste ironie de l'histoire - a été gravement entachée par l'accusation de faits de torture portée contre des membres du Tuscania ${ }^{37}$.

33. Autrement dit, le message est clair : « sans les carabiniers, l'Italie ne pourrait jamais être crédible dans le cadre des opérations extérieures ».

34. Servizio per le Informazioni e la Sicurezza Militare.

35. Reparto Operativo Speciale.

36. Sur 208 arrestations de suspects terroristes vantées par Berlusconi, la grande majorité a concerné des personnes absolument innocentes, de simples immigrés originaires de pays considérés comme islamistes (parmi eux 27 Pakistanais, 17 Afghans et des dizaines de Maghrébins et d'Egyptiens - voir nos rapport pour le programme de recherche Challenge et les travaux de Bonini C., D’Avanzo G., op. cit.).

37. Le 5 juin 1997, l'hebdomadaire Panorama publie un reportage, photos à l'appui, portant sur les brutalités commises par des militaires italiens en Somalie. Le 8 août 1997, la Commission d'enquête nommée par le gouvernement affirme avoir les preuves de trois cas sur huit signalés, mais exclut toute responsabilité de la hiérarchie militaire. Le 22 février 2001, la Cour d'appel de Florence classe en prescription le délit d'abus d'autorité dont était accusé un maréchal des parachutistes qui avait été photographié pendant qu'il torturait - par décharges électriques - un Somalien lors de la mission Ibis, à Mogadiscio en 1997. 
D'ailleurs, dans le cadre de cette même mission, un certain nombre de militaires canadiens et d'autres pays ont été inculpés pour le même type de délits ${ }^{38}$. Mais, lors du procès au tribunal de Livourne en 1998, plusieurs soldats, sousofficiers et officiers du bataillon Tuscania ont été acquittés, bien que les faits incriminés se fussent révélées véridiques : selon le tribunal, aucun militaire en particulier n'a pu être identifié ${ }^{39}$. De plus, selon certains journalistes ${ }^{40}$, des officiers du Tuscania connaîtraient l'identité des responsables de l'assassinat des journalistes Ilaria Alpi et Miran Hrovatin le 20 mars 1994 en Somalie 41. Ces assassinats auraient été perpétrés à la fois parce que ces journalistes enquêtaient en Somalie dès 1993 sur des trafics d'armes, de drogues et de déchets radioactifs organisés par des Italiens et des «mercenaires » somaliens, et peutêtre surtout parce qu'ils auraient photographié les actes de torture impliquant certains militaires du Tuscania ${ }^{42}$. La justice italienne n’a, à ce jour, pas réussi à découvrir les responsables et les mandants, malgré nombre d'éléments susceptibles de permettre leur identification.

Pour autant, les missions extérieures des carabiniers n'ont pas impliqué leur désengagement des missions de sécurité intérieure, loin s'en faut. On peut sans doute s'étonner du fait que le bataillon Tuscania et les mêmes militaires qui avaient été en Somalie aient pu jouer un rôle de premier plan lors des violences policières à l'occasion du sommet du G8 à Gênes (20 juillet 2001), et en particulier dans la mort de Carlo Giuliani. Il pourrait y avoir là une démonstration éclairante de la portée des missions militaires à l'étranger en tant qu'expériences « révolutionnaires », car hybrides, entre militaire et policier.

Le lieutenant-colonel Truglio commandait les « Compagnies d'intervention résolutive » (CCIR) créées spécialement pour le sommet de Gênes du G8 avec, à ses cotés, le capitaine Cappello ${ }^{43}$. Ces cinq compagnies spéciales, dirigées par des officiers entraînés à la guerre, correspondaient au type de gestion militaro-policière caractéristique des missions militaires en externe. Ainsi, lors de la 40e audience du procès faisant suite aux « événements » relatifs au sommet du G8,

38. Voir notamment Brodeur J.-P., « Maintien et imposition de la paix en Somalie (1992-1995) », Cultures \& Conflits, n²9-30, 1998, pp. 175-193.

39. Voir Amnesty International, Italy: A briefing for the UN Committee against Torture (AI Index : EUR 30/02/99) et L'Italia e i diritti umani, Rome, Section italienne d'AI, juin 2000.

40. www.ilariaalpi.it

41. Le cas de l'assassinat de ces reporters est assez connu et a fait l'objet d'une enquête parlementaire sous le gouvernement Berlusconi, qui a abouti à l'étouffement de l'affaire. Il s'agissait d'enquêter à la fois sur le trafic d'armes, de déchets et sur les comportements illicites des carabiniers et militaires italiens (pour une documentation détaillée voir le site Internet cité en note 40).

42. http://www.ilariaalpi.it/index.php?id_sezione=3\&id_notizia $=317$

43. Voir en particulier les articles d'A. Mantovani sur le manifeste («il manifesto ») du 29 décembre 2002. Pour une documentation plus vaste sur les faits du G8 de Gênes voir Gubitosa C., (dir.), Nome per nome, livre et CD, Berti éditeur, supplément au n ${ }^{\circ} 41$ d'Altreconomia, T2 06045895 ; Palidda S., « Las mutations de la gestion pacifica y negoociada de la protesta social », Contrapoder, 10/2007, pp. 38-58; voir la documentation sur les sites http://www.processig8.org/ et http://www.supportolegale.org/) des comités « Piazza Carlo Giuliani » et « Verità e Giustizia per Genova », et le dvd récent : OP Genova, 2001 (en anglais, français, allemand, espagnol et italien). 
le lieutenant Mirante des CCIR n'a pas hésité à affirmer que ce qui s'était passé à Gênes rappelait la Première Guerre mondiale car la « guerre et l'ordre public sont la même chose 44 ». Il a également cité Sun Tsu et rappelé, à propos de la mort de Giuliani, qu'il avait vu beaucoup de morts tout au long de sa carrière militaire.

Selon la plus récente reconstruction des faits au cours du procès, les choix opérationnels des carabiniers lors du sommet de Gênes semblent avoir suivi la volonté du G8 : arrêter l'élan qui, de Seattle à Nice, de Prague à Göteborg, avait alimenté un mouvement mondial de plus en plus insupportable pour une forme de domination globale libre d'agir comme elle l'entend ${ }^{45}$. Ainsi, comme le montrent les reportages filmés par un certain nombre de médias ${ }^{46}$, les chars du Tuscania ont été lancés contre la foule et, selon les rapports du Tuscania luimême, il y eut pas moins de 18 coups de feu tirés par les carabiniers sur la place où Giuliani a été tué. De son côté, la police d'Etat a semblé chercher à concurrencer ses collègues carabiniers par son unité spéciale anti-subversive. Dans les discussions téléphoniques entre dirigeants des polices auditionnées lors du procès, on entend plusieurs fois l'ordre de "faire des prisonniers ", langage plus représentatif d'une opération militaire que d'une opération policière.

Une rapide analyse des trajectoires individuelles des auteurs présumés des «faits de Gênes » paraît pourtant indiquer que ces derniers n'ont pas semblé particulièrement pénalisants pour eux. Ainsi, à la suite du sommet de Gênes, le lieutenant-colonel Truglio s'est vu confier la salle opérationnelle centrale du commandement général des carabiniers, l'un des principaux nœuds de circulation des informations à l'intérieur de l'armée ${ }^{47}$. Il est alors difficile d'affirmer que son rôle dans l'expérimentation de la militarisation de la gestion de l'ordre public (que l'on pourrait analyser comme "révolution policière ", l'équivalent, dans la police, de la RMA ${ }^{48}$ ) n'ait pas été avalisé par les niveaux hiérarchiques supérieurs ${ }^{49}$. L'officier des carabiniers qui, à Gênes, coordonnait les opérations depuis le quartier général de la Foire de la mer (lieu d'installation

44. Transcription de l'audience du 15 mars 2005. Voir :

http://www.supportolegale.org/?q=node/142

45. Selon Condoleeza Rice lors de son audition sur le 11 septembre 2001, il fallait intervenir avec force à Gênes car on craignait la présence de terroristes parmi les manifestants qui voulaient attaquer la zone rouge. Autrement dit, il a fallu traiter les manifestants comme des ennemis de guerre. Voir Dal Lago A., " Genova, origine della crisi politica », Il Manifesto, 7 juillet 2007 et Polizia globale, Vérone, Ombrecorte, 2003.

46. Voir le récent DVD : OP Genova 2001, produit avec le soutien du Genoa Legal Forum. Sur les sites : https://www.supportolegale.org/, http://www.veritagiustizia.it/ et http://www.piazzacarlogiuliani.org/, on peut consulter de nombreux documents du procès et visionner des enregistrements vidéos dans plusieurs langues.

47. Rappelons que tous les dirigeants et agents des forces de polices en procès pour les diverses violences commises contre les manifestants ont obtenu des promotions de carrière. Par ailleurs, le chef de police qui est soupçonné d'avoir couvert ces comportements et poussé certains à des faux témoignages, est maintenant devenu chef du cabinet du ministre de l'Intérieur.

48. Palidda S., Revolution in Police Affairs, à paraitre.

49. Voir notamment Dupont B., Lemieux F. (dirs), La Militarisation des appareils policiers, Laval, Les Presses de l'université de Laval, 2005. 
des forces de police) le colonel Leonardo Leso (supérieur de Truglio) est, quant à lui, devenu peu de temps après général et passé au commandement de la Seconde Brigade mobile des carabiniers qui réunit le Tuscania, les « têtes de cuir » du GIS 50 (Groupe d'intervention spéciale pour la lutte contre terrorisme) et deux autres régiments spéciaux. Leso avait, avant le sommet de Gênes, commandé le MSU (Multinational specialized Unit) - l'unité « gendarmique » de l'OTAN formée surtout par et à l'initiative des carabiniers italiens - en Bosnie-Herzégovine, et il appartient au cercle restreint des militaires décorés par la Legion of merit, la plus haute distinction que le président des Etats-Unis peut accorder à un étranger. Pourtant, selon le témoignage du sénateur Malabarba lors du procès pour les «faits de Gênes ", à la Foire au moment des manifestations, circulaient sous les yeux de Leso des groupes de manifestants francophones et germanophones semblables aux black blocs; apportant ainsi un certain crédit aux soupçons de nombre d'observateurs et avocats qu'une partie des black blocs était composée de provocateurs qui auraient été recrutés, en particulier parmi les hooligans « naziskins » par, ou du moins avec, l'aval des carabiniers ${ }^{51}$.

On ne saurait cependant en rester au constat d'une simple « importation » à sens unique, de pratiques de type « guerrier » en interne. L'étude des trajectoires de ces acteurs au sein des carabiniers montre en effet également que leur investissement professionnel dans les opérations extérieures n’a jamais cessé : Truglio, Cappello, Leso ainsi que leurs hommes du Tuscania sont passés des missions en Somalie à celles en Afghanistan, sans oublier la BosnieHerzégovine, le Kosovo, l'Albanie et l'Irak. Il est à cet égard également assez révélateur que, lors de la mission en Irak, le Tuscania ait été chargé entre autres tâches, de former les policiers irakiens dans la gestion du désordre public et que, lors de ces formations, l'enregistrement vidéo de l'intervention lors des manifestions à Gênes ait été visionné 52 .

La valorisation des succès des carabiniers depuis les années 1990 dans les missions militaires à l'étranger a contribué à un autre résultat important : la localisation du quartier général de la force de gendarmerie européenne (EuroGendFor ou FGE) à Vicenza. La FGE a été créée le 17 septembre 2004 à Noordwijk, aux Pays-Bas ${ }^{53}$. Née d'une initiative et d'une proposition française,

50. Gruppo Intervento Speciale.

51. Voir les articles de A. Mantovani dans Il Manifesto du 29 décembre 2002.

52 . Voir : http://www.jgcinema.org/pages/view.php?cat=articoli_dossier\&id=163\&id_film=0\&id_ dossier=18; l'emploi de l'enregistrement vidéo des événements de Gênes pour former les policiers irakiens a été confirmé par Il Manifesto et par Repubblica le $1^{\mathrm{er}}$ octobre 2004, qui citent des sources fiables internes aux forces armées.

53. Les hommes de la FGE pourront êtres déployés dans les trente jours, y compris au-delà des frontières de l'Union. Leurs missions concourront à « la gestion de crise » et relèvent à la fois du maintien de l'ordre et des activités de police judiciaire pour lutter contre le terrorisme ou le crime organisé. Elle a été déclarée opérationnelle le 20 juillet 2006.

Voir : http://www.defense.gouv.fr/gendarmerie/decouverte/missions/internationales/presence _en_europe/eurogendfor_la_force_de_gendarmerie_europeenne 
elle a bénéficié de la grande disponibilité de l'Italie et en particulier des carabiniers ${ }^{54}$. Le commandement suit une rotation par pays et, en 2006, il a été notamment assuré par le général Leso. Par ailleurs le Coespu, le nouveau corps des carabiniers intégré au FGE, a été mis en place et commandé par le général Leso. Rappelons aussi qu'à Saint-Astier, en France, du 15 au 17 juin 2005, se sont déroulés les premiers exercices d'entraînement des futurs gendarmes européens avec des exercices anti-émeutes en milieu urbain : une formation représentative d'une force hybride dont les compétences vont de la sécurité intérieure à la sécurité globale; en somme, une sorte de prototype de "police globale ». Sa fonction a été explicitée par un porte-parole du Conseil de l'Union européenne :

«Ce qui ressort de l'expérience en matière de gestion de sortie de crise internationale, c'est que l'on a besoin d'un personnel différent de la police civile et capable de mener une tâche qui ne soit pas strictement militaire. Au Kosovo, on s'est rendu compte du fait que l'on avait besoin de forces capables de gérer les émeutes par exemple 55 ».

Les opérations extérieures contemporaines ont ainsi permis aux carabiniers de valoriser leurs domaines de compétences militaro-policières, ce à quoi ils s'attèlent précisément au sein du FGE. Cela est d'autant plus le cas que les carabiniers se spécialisent également dans la formation de policiers. Par exemple, à Mitrovica des gendarmes italiens et français auraient "permis l'accompagnement et la transition naturelle » en aidant à former les polices locales. De la même façon, lorsqu'en Irak il s'est révélé que l'administration américaine n'avait rien prévu pour assurer la transition une fois la phase proprement militaire passée, c'est à cela - au dire des commandants du Tuscania - que cette dernière a travaillé.

Enfin, le rôle de plus en central du type de savoir-faire revendiqué par les carabiniers a notamment été confirmé par le plan d'action du G8 du 10 juin 2004 afin de "développer les capacité mondiales de maintien de la paix». Celui-ci prévoit la formation et l'entrânement, avant 2010, d'environ 75000 peacekeepers internationaux dont $10 \%$ seront composés par des effectifs du genre carabiniers/gendarmerie ${ }^{56}$. Ainsi, les Stability Police Units (SPU) prévus par ce plan, des unités flexibles et adaptables combinant voire hybridant tâches militaires, policières et civiles ${ }^{57}$, requérront précisément les qualités dont les carabiniers italiens se proclament dotés. Les reconnaissances de ces qualités aux carabiniers ne manquent d'ailleurs pas : sur le site du consulat américain de Milan on trouve le titre «Les Etats-Unis honorent les nouveaux diplômés du Centre

54. La FGE regroupe les forces de police à statut militaire des cinq pays de l'Union européenne qui en disposent : la garde civile espagnole, la gendarmerie française, les carabiniers italiens, la maréchaussée royale des Pays-Bas et la garde nationale républicaine portugaise (d'autres pays européens ont fait acte de candidature, l'Allemagne s'y est refusée).

55 . http://www.europeplusnet.info/article422.html

56 . http://www.carabinieri.it/Internet/Arma/CoESPU/

57 . http://www.globalproject.info/art-7051.html 
d'excellence pour les Stability Police Units ", accompagné des discours du général Leso et du général David Bustamante, le Senior Officer in Charge 58.

\section{La continuité avec les gouvernement de gauche}

L'importance des missions militaires à l'étranger pour l'Italie est telle que celles-ci jouissent du soutien quasi unanime du Parlement, mais aussi de pans entiers de l'opinion publique. L'article 11 de la Charte constitutionnelle semble ainsi dépassé dans un pays qui se considère comme l'une des cinq premières puissances $\mathrm{du}$ "monde globalisé » et qui se vante, par conséquent, d'avoir partout des intérêts à défendre.

Ainsi, le gouvernement Prodi s'est montré très zélé dans le respect des accords passés avec les Etats-Unis, notamment en ce qui concerne le bouclier spatial et la production militaire (voir infra), et il s'est engagé à soutenir une mission encore plus importante en Afghanistan ainsi qu'une autre, nouvelle, au Liban.

En même temps, le retrait de l'Irak en 2006 a rapidement été compensé par le financement de 3498000 euros pour payer la SMP qui, en Irak, protégera les Italiens et leurs intérêts ${ }^{59}$ - notamment les techniciens de l'ENI et quelques représentants d'ONG italiennes - présents en Irak au titre de «Sécurité d'Ur », une équipe provinciale de reconstruction (Provincial Reconstruction Team; PRT South East) créée en 2006 dans la province irakienne de Dhi Qar. La SMP britannique, Aegis Defence Services, choisie par le gouvernement Prodi est présente en Irak depuis 2004, après avoir établi un contrat de 293 millions de dollars avec le Pentagone. Son président, Tim Spicer, a pourtant été impliqué dans un certain nombre de scandales politiques. Ceux-ci étaient liés notamment à l'intervention en 1997 de sa précédente SMP, Sandline International, sur l'île de Bougainville (Papouasie - Nouvelle-Guinée), et au "Arms-to-Africa Affair ", également en 1997, à la suite de la révélation selon laquelle des armes avaient été livrées par l'entreprise, avec la bénédiction de l'Ambassade britannique sur place, au Sierra Leone en violation d'un embargo de l'ONU sur les exportations d'armes vers ce pays en pleine guerre civile ${ }^{60}$. Malgré le retrait italien en Irak, un dirigeant d'une ONG («Un Ponte per») a affirmé récemment qu’il était étonnant qu’en Irak, il y ait encore besoin d'une «protection privée» des Italiens, les Provincial Reconstruction Teams (PRT) devant être sous la tutelle des militaires américains. Cependant, au vu du grand nombre d'enlèvements récents, le problème de la protection des civils italiens à l'étranger a pris une importance particulière en Italie ${ }^{61}$.

58 . http://milan.usconsulate.gov/news/NE_ITA_022406_COESPU.htm

59. Voir article de Gualco M., "Iraq, nel ddl tre milioni di euro per i “contractors" ", L'Unità, 16 mars 2007 : www.unita.it, http://www.resistenze.org/sito/os/ip/osip7c17-001249.htm

60. Voir notamment Singer P.W., Corporate Warriors. The Rise of the Privatised Military Industry, New York, Cornell University Press, 2003 ; Chapleau P., Misser F., Mercenaires SA, Paris, Desclée de Brouwer, 1998.

61. Notamment les personnels de l'ENI au Niger. 
Après deux ans de légère diminution des ressources allouées à la Défense par le gouvernement Berlusconi, qui avait établi ce budget à 18862 millions d'euros en 2006, le gouvernement Prodi a fixé les dépenses italiennes pour la Défense à 21011 millions d'euros en 2007. Celui-ci sera de 23352 millions d'euros en 2008 (dont environ un quart pour les carabiniers) 62. En d'autres termes, on peut constater une augmentation de $11,1 \%$ entre 2007 et $2008{ }^{63}$.

Concernant les missions à l'étranger, le pays confirme sa présence dans 19 pays et 27 missions, avec la participation de 7714 militaires. Parmi ces pays, on trouve l'Afghanistan (mission ISAF, 2290 hommes et femmes), le Liban (mission UNIFIL, environ 2400 hommes et femmes) et les Balkans (Bosnie, Kosovo, Macedoine, ce qui représente un peu plus de 2600 effectifs). La mission en Afghanistan coûte un peu plus de 310 millions d'euros chaque année.

Comme en 2007, le budget de 2008 prévoit pour ces missions un milliard d'euros, accompagné de l'automatisme du re-financement des missions sans besoin de passer tous les six mois par le vote parlementaire ${ }^{64}$. Par ailleurs, la programmation du gouvernement Prodi prévoit notamment : 121 avions de chasse EF2000, 22 avions de transport de troupes et matériels C-130J ; un programme de construction de superchasseurs américains JSF dont l'Italie s'est engagée à acheter une centaine ; 116 hélicoptères $\mathrm{NH}-90$; 4 sous-marins ; 12 navires frégates ; environ 920 blindés et chars; un second porte-avion ; 70 canons d'artillerie 65 .

Suite à la décision d'installer la 173e Brigade aérotransportée américaine en Italie pour constituer une brigade «combat team », se construit à Vicence une nouvelle base, qui va provoquer une vaste mobilisation de la population locale 66. Selon le rapport du Pentagone Base Structure Report 2006, les forces

62. Voir www.governo.it, « Legge finanziaria 2008-2010» et www.sbilanciamoci.org. En 2008, 155 millions d'euros sont destinés aux frégates Freem et 968 millions aux intercepteurs Eurofighter. Remarquons que l'article 93, au nom d'exigences liées au maintien de l'ordre public, prévoit un plan d'embauche de 50 millions d'euros pour 2008, 120 millions pour 2009 et 140 millions pour 2010. L'article 94 prévoit la possibilité de mutation temporaire d'effectifs de l'armée de terre, de la marine et de l'armée de l'air vers les forces de police à ordonnancement civil et militaire.

63. Selon le SIPRI en 2007, les dépenses militaires en 2006 ont été les suivantes : Etats-Unis : 528,7 milliards de dollars ; Royaume-Uni : 59,2 ; France : 53,1; Chine : 49,5 ; Japon ; 43,7; Allemagne : 37,0; Russie : 34,7 ; Italie : 29,9; Arabie Saoudite : 29,0; Inde : 23,9. Dans les dépenses par habitant, l'Italie passe de 468 dollars en 2005, à 514 en 2006 (l'Allemagne arrive à 477 dollars).

64 . La loi financière adoptée par le gouvernement Prodi affirme ainsi : «Pour 2007, 2008 et 2009, la dépense de jusqu'à 1 milliard d'euros pour le financement de la participation italienne aux missions internationales de paix est autorisée ». En réalité, selon des pacifistes (http://www.peacereporter.net/dettaglio_articolo.php?idpa=\&idc=73\&ida=\&idt=\&idart=6405), les sommes allouées à ces missions seraient plus importantes car certaines seraient « cachées » dans d'autres chapitres, notamment celui de la modernisation des instruments militaires, celui de la recherche et développement pour les missions, etc.

65. Pour plus de détails, voir l'analyse d'Alberto Stefanelli sur : http://www.mercatiesplosivi.com/guerrepace/134Stefanelli.htm

66. Cette mobilisation pose de sérieux problèmes au centre-gauche du gouvernement car elle est 
armées américaines en Italie possèdent 1546 immeubles et en ont 1168 autres en location, ce qui représente une surface totale de presque 2 millions de $\mathrm{m}^{2}$. Dans l'Allied Contributions To The Common Defense publié par le Pentagone, l'Italie figure parmi les premiers contributeurs au maintien des bases américaines (à la hauteur de 41 \% soit l'équivalent de 366,54 millions de dollars en 2003), alors que pour le Royaume-Uni ce taux est de $27 \%$, pour l'Allemagne de $32 \%$ et pour les pays européens, il est en moyenne de $28 \% 67$.

Ainsi, l'Italie se dote d'armements stratégiques, mais toujours en tant qu'allié subordonné des Etats-Unis et toujours au nom d'un devoir d'engagement dans des «missions de paix ». L'engagement zélé du centre-gauche dans les affaires militaires (ainsi que dans les affaires policières) apparaît aujourd'hui plus important que celui adopté par le centre-droit de Berlusconi. En effet, ce dernier avait surtout privilégié ses affaires personnelles. Le centre-gauche semble alors davantage influencé par des intérêts militaires et policiers ainsi que par ceux liés aux délocalisations de nombreuses activités économiques (parmi lesquels il ne faut pas oublier les syndicats des travailleurs de l'industrie militaire, les fonctionnaires des partis et les parlementaires dans les conseils d'administration des sociétés qui fournissent les forces armées et les polices en matériel).Malgré le retrait de l'Irak, l'Italie connaît donc en 2007 une augmentation des effectifs à l'étranger - et en particulier des carabiniers - parallèlement à une augmentation des effectifs policiers pour la sécurité intérieure.

le fait de ses électeurs. Par ailleurs, des intellectuels de gauche et des anciens leaders démocrates-chrétiens ont exprimé des doutes sur la nécessité de cette base. Ainsi, Andreotti a-t-il déclaré : « désormais l'Union soviétique n'existe plus. Alors pourquoi cette base et des structures militaires de ce genre? En tout cas pas pour se défendre contre le terrorisme ", Corriere della Sera, 2 mars 2007. Voir : http://www.nodalmolin.eu/

67. Voir le rapport « La finanziaria per noi » sur le site http://www.sbilanciamoci.org/ qui, chaque année, réunit un grand nombre d'associations et d'experts pour analyser la loi des finances et proposer des alternatives, sans toutefois rompre avec les intérêts économiques et sociaux du pays. 